\title{
Pleiotropic effects of fenretinide in neuroblastoma cell lines and multicellular tumor spheroids
}

\author{
ROOS CUPERUS ${ }^{1}$, GODELIEVE A.M. TYTGAT ${ }^{1,3}$, RENÉ LEEN $^{1}$, PEDRO BRITES ${ }^{1}$, \\ JOHANNES BRAS ${ }^{2}$, HUIB N. CARON ${ }^{1}$ and ANDRÉ B.P. VAN KUILENBURG ${ }^{1}$
}

${ }^{1}$ Laboratory Genetic Metabolic Diseases and Department of Pediatrics/Emma Children's Hospital, ${ }^{2}$ Department of Pathology, Academic Medical Centre, University of Amsterdam, P.O. Box 22700, 1100 DE Amsterdam; ${ }^{3}$ Department of Pediatrics, Zaans Medical Centre, P.O. Box 210, 1500 EE Zaandam, The Netherlands

Received November 6, 2007; Accepted December 13, 2007

\begin{abstract}
The efficacy and mechanism of action of fenretinide (4-HPR), a vitamin A analogue, was investigated in a panel of six neuroblastoma cell lines and multicellular tumor spheroids. The latter are three dimensional cell aggregates and as such, a model for micrometastases. In all cell lines, the production of reactive oxygen species (ROS) increased with $163-680 \%$ after $1 \mathrm{~h}$ of treatment with 4-HPR. In addition, a decrease of the mitochondrial membrane potential of $30-75 \%$ was observed after $4 \mathrm{~h}$ of incubation with 4-HPR. A 6-12-fold difference was observed between the $\mathrm{IC}_{50}$ values for cell proliferation and viability between the most sensitive (IMR32) and most resistant (NASS) cell line towards 4-HPR. Flow cytometric analysis showed an increased amount of apoptotic bodies and no cell-cycle arrest. The antioxidant Trolox completely inhibited the accumulation of 4HPR-induced ROS and prevented the 4HPR-associated cytotoxicity. In all neuroblastoma spheroids, 4-HPR induced a complete cytostasis at clinical relevant concentrations $(3-10 \mu \mathrm{M})$. Immunohistochemical analysis of 4-HPR-treated spheroids showed a decreased staining for proliferation marker Ki-67 and an increased staining for cleaved-PARP, a marker of apoptosis. Our results suggest that 4-HPR might be a promising agent for the treatment of micrometastases and high-risk neuroblastoma.
\end{abstract}

\section{Introduction}

Neuroblastoma is the most common solid extracranial tumor in children. The prognosis for children suffering from neuro-

Correspondence to: Dr André B.P. Van Kuilenburg, Laboratory Genetic Metabolic Diseases and Department of Pediatrics/Emma Children's Hospital, Academic Medical Centre, University of Amsterdam, P.O. Box 22700, 1100 DE Amsterdam, The Netherlands E-mail: a.b.vankuilenburg@amc.uva.nl

Key words: neuroblastoma, fenretinide, spheroids, mitochondrial membrane potential, oxidative stress, apoptosis blastoma is highly dependent on the age at diagnosis and the stage of the disease. Patients suffering from metastasized neuroblastoma with amplification of the MYCN oncogene, which is found in approximately $25 \%$ of primary, predominantly metastasized neuroblastomas, have a very poor prognosis (1). Current therapy is based on treatment with chemotherapeutic drugs, surgery, treatment with ${ }^{131} \mathrm{I}$-metaiodobenzylguanidine ( $\left.{ }^{131} \mathrm{I}-\mathrm{MIBG}\right)$ and autologous stem cell transplantation. Despite these aggressive therapeutic strategies, the likelihood for survival for patients suffering from highrisk neuroblastoma has not increased significantly over the last 15 years $(2,3)$. Therefore, new and effective therapeutic strategies are being developed to improve prognosis in these patients.

Stage $4 \mathrm{~s}$ neuroblastoma is characterized by spontaneous regression of the tumor by differentiation. Retinoids are essential regulators of cell growth, differentiation and cell death (4). All-trans retinoic acid (ATRA) and 13-cis retinoic acid (13-cis-RA) have been shown to induce differentiation and death in neuroblastoma cells (5). Although treatment with 13-cis-retinoic acid increased the event-free survival in children with advanced-stage neuroblastoma (6), its use has been restricted by dose-limiting side effects (5) and increased resistance of retinoic acid differentiated cells to chemotherapeutic drugs (7). Fenretinide (N-(4-hydroxyphenyl) retinamide) (4-HPR) is a synthetic retinoic acid derivate which induced apoptosis rather than differentiation in a variety of tumors (8-11).

The precise mechanism underlying the apoptosis-inducing properties of 4-HPR is not yet fully understood. It has been suggested that 4-HPR can induce apoptosis by both retinoic acid receptor (RAR)-dependent and reactive oxygen species (ROS)-dependent pathways (12-15). Mitochondrial membrane depolarization, which plays a key role in the process of apoptosis, has been observed in some tumor types treated with 4-HPR (16). However, it has been reported that 4-HPR did not alter the mitochondrial membrane potential $\left(\Delta \Psi_{\mathrm{m}}\right)$ in neuroblastoma (17).

It is known that solid tumors require adequate diffusion of nutrients for tumor growth. Spheroids are three dimensional aggregates of cancer cells that, due to their cellular organization, have been shown to resemble in vivo tumors with 
respect to growth rates and sensitivity towards chemotherapeutic drugs (18). Most importantly, they resemble micrometastases during the avascular phase of their development (18), which provides the opportunity to study phenomena such as the penetration and effectiveness of cytotoxic agents on spheroid size. So far, the effect of 4-HPR has not been studied on a panel of neuroblastoma multicellular spheroids combined with immunohistochemical evaluation. In this report, we describe the effect of 4-HPR on various biological parameters in a panel of $M Y C N$-single copy and $M Y C N$ amplified neuroblastoma cell line monolayers as well as spheroids.

\section{Materials and methods}

Chemicals. 4-HPR (Sigma, St. Louis, MO, USA), was dissolved in $100 \%$ ethanol, stored at $4^{\circ} \mathrm{C}$ and protected from light. Serial dilutions were prepared from the stock solution with growth medium just before use. $\mathrm{H}_{2} \mathrm{O}_{2}$ (Sigma) was prepared as a $100 \mu \mathrm{M}$ stock solution in double distilled $\mathrm{H}_{2} \mathrm{O}$ immediately before use. Trolox (Sigma) was prepared as a $0.5 \mathrm{M}$ stock solution in $100 \%$ ethanol and kept at $-20^{\circ} \mathrm{C}$ for one month.

Cell culture. Three MYCN single copy neuroblastoma cell lines (FISK, NASS, SY5Y) and three MYCN amplified neuroblastoma cell lines (IMR32, SJ8, SJNB10) were cultured in RPMI-1640 culture medium (Gibco, Invitrogen, Carlsbad, CA, USA) supplemented with $10 \%$ heat inactivated fetal bovine serum, $50 \mathrm{U} / \mathrm{ml}$ penicillin/streptomycin and $4 \mathrm{mM}$ glutamine. Cells were grown at $37^{\circ} \mathrm{C}, 5 \% \mathrm{CO}_{2}$ in $95 \%$ humidified air, all culture flasks and plates were from Corning (Corning, NY, USA). All cell lines were a generous gift of Professor R. Versteeg (Department of Human Genetics, Academical Medical Centre, Amsterdam).

Spheroids were prepared by growing 400,000 cells in $4 \mathrm{ml}$ culture medium in an ultra-low attachment sterile Petri dish. Because the cells could not adhere to the bottom of the dish they cohered, forming small aggregates. After three days, small spheroids $(0.3-0.5 \mathrm{~mm})$ were formed and these were individually transferred to ultra-low attachment 24-well plates. One well contained one spheroid in $1 \mathrm{ml}$ culture medium. Spheroids were treated with different concentrations 4-HPR for three weeks, without changing the culture medium. The experiments were performed in quadruplicate. Spheroid size was monitored weekly by measurement of the cross-sectional area of individual spheroids five-times magnified photos using a microscope (19) (microscope: Leica DMIL, Wetzlar, Germany. camera: SV camera, Lambert instruments, Leutingewolde, The Netherlands, Image Pro Plus 4.1).

Measurement of cell viability and proliferation. Cells were plated in 96-well plates at a density of 5,000 or 6,500 cells per cell line per well in a total volume of $100 \mu \mathrm{l}$ and were allowed to adhere overnight, after which the medium was replaced by medium containing different concentrations 4-HPR $(0-40 \mu \mathrm{M})$. After 24, 48 and $72 \mathrm{~h}$ medium with 4-HPR was changed for fresh medium and the viability of the cells was measured with MTS assay (Promega, Madison, WI, USA) according to the manufacturer's protocol. MTS incubation lasted for $4 \mathrm{~h}$ at $37^{\circ} \mathrm{C}$. The effect of the antioxidant trolox on viability was investigated by incubating the cells with 4-HPR (5 and $10 \mu \mathrm{M}$ ) and/or trolox $(500 \mu \mathrm{M}$ ) for $72 \mathrm{~h}$ followed by the MTS assay. Cell proliferation was measured using a 5-bromo-2'-deoxyuridine (BrdU) incorporation assay (Roche Applied Science, Penzberg, Germany), according to the manufacturer's instructions. Cells were incubated for $2 \mathrm{~h}$ at $37^{\circ} \mathrm{C}$ with BrdU labeling solution, without refreshing the medium. The experiments were performed in quadruplicate. The dose-absorption curves were used to derive the $\mathrm{IC}_{50}$ values.

Cell cycle analysis. Apoptosis was evaluated by flow cytometry of propidium iodide (PI) stained cells. Cells $(500,000)$ were grown in $4 \mathrm{ml}$ culture medium in a $25 \mathrm{~cm}^{2}$ flask and treated with 4-HPR (1-5 $\mu \mathrm{M})$ for 24, 48 and $72 \mathrm{~h}$. Both attached and floating cells were harvested and fixed in $70 \%$ ethanol in PBS and stored at $4{ }^{\circ} \mathrm{C}$. For flow cytometric analysis samples were centrifuged at $1000 \mathrm{x}$ g for $1 \mathrm{~min}$. Cell pellets were resuspended in $200 \mu \mathrm{l}$ PBS after which $200 \mu 1$ RNAse-PBS in a $4-\mathrm{mg} / \mathrm{ml}$ concentration was added. Cells were stained with $400 \mu \mathrm{l}$ PI-saponin $(0.05 \mathrm{mg} \mathrm{PI} / \mathrm{ml} 0.02 \%$ (w/v) (saponinPBS), vortexed thoroughly and incubated for $10 \mathrm{~min}$ at $37^{\circ} \mathrm{C}$. The stained cell nuclei were analyzed by a FACS Calibur flow cytometer (Beckton-Dickinson) and the data were analyzed using WinMD1 version 2.8 software.

Measurement of apoptosis - PARP analysis. Cells $(750,000)$ were cultured in $4 \mathrm{ml}$ in a $25 \mathrm{~cm}^{2}$ flask and allowed to adhere overnight, after which the medium was replaced by medium containing 4-HPR $(0-20 \mu \mathrm{M})$. After $24 \mathrm{~h}$, both attached and floating cells were collected and centrifugated and the pellet was resuspended in PBS, sonicated $(2 \times 20 \mathrm{~s}, 8 \mathrm{~W})$ and boiled ( 5 min). Protein concentrations were detected using a BCA protein determination assay (20). Samples were diluted to equal protein concentrations in sample buffer.

Sodium dodecyl sulphate-polyacrylamide gel electrophoresis was performed as described by Laemmli (21) followed by Western blotting using the primary mouse anti-human poly (ADP-ribose) polymerase (PARP) monoclonal antibody 1:10,000 diluted (Biomol, UK) and rabbit anti-mouse secondary antibody in a 1:5,000 dilution in PBS containing $3 \%$ BSA and $0.01 \%$ Tween-20 conjugated with horseradish peroxidase. After washing the membrane, detection of PARP was performed with enhanced chemiluminescence. Equal loading was confirmed using Ponceau S staining (22). Neuroblastoma cells treated with the histone deacetylase inhibitor BL1521 were used as a positive control (23).

Immunohistochemistry. Spheroids were immersion fixed in buffered formalin and embedded in paraffin. Dewaxed and rehydrated sections $(4.5 \mu \mathrm{m}$ thick) were stained with hematoxylin and eosin (H\&E) for morphology. Endogenous peroxidases were blocked with $3 \%$ hydrogen peroxide. After antigen retrieval (microwaving, Tris-EDTA) aspecific binding was prevented by blocking with 5\% normal goat serum. Apoptosis was detected using anti-cleaved-PARP 1:200 (BD Biosciences, USA), proliferation was detected using anti-Ki-67 1:200 (Dako, M7240), both incubated $1 \mathrm{~h}$ at room temperature. Sections were blocked using post-antibody 
blocking (Immunologic) for $15 \mathrm{~min}$ at room temperature. Sections were incubated with biotinylated secondary poly-HPR anti-rabbit/mouse antibodies and avidin-biotin complex (Vectastain ABC Kit). Immunoreactivity was visualized by incubation with 3,3'-diaminobenzidine (Sigma), tissues were counterstained with hematoxylin QS (Vector laboratories, USA). Sections were dehydrated, cleared in HistoClear II and mounted in DPX mounting for histology (Fluka, St. Louis, MO, USA). For negative controls primary antibodies were omitted. Sections were examined using a Zeiss microscope and photographed using a Leica camera.

Reactive oxygen species (ROS). ROS production was detected using $\mathrm{CM}-\mathrm{H}_{2}$ DCFDA (Invitrogen, Molecular Probes). Cells $(100,000)$ were cultured in $1 \mathrm{ml}$ medium in 12 -well plates and allowed to adhere overnight. Subsequently, cells were treated with a concentration range of 4-HPR $(0-10 \mu \mathrm{M})$ for 1 , 2, 3, 6 and $24 \mathrm{~h}$. The ROS responsive dye CM- $\mathrm{H}_{2}$ DCFDA was prepared in DMSO in a $5-\mu \mathrm{M}$ stock solution and kept on ice until use. Culture medium was replaced by $0.8 \mathrm{ml}$ freshly prepared $\mathrm{CM}-\mathrm{H}_{2}$ DCFDA-solution and incubated at $37^{\circ} \mathrm{C}$ for $15 \mathrm{~min}$. Subsequently, fluorescence was measured in a microplate reader (BMG Labtech, FLUOstar Optima); excitation $485 \mathrm{~nm}$, emission $520 \mathrm{~nm}$. Cells were harvested by trypsinization and protein determination was performed using bicinchoninic acid (BCA) reagens (Sigma) according to the manufacturer's protocol using bovine serum albumin (BSA) as a standard. ROS production was also measured after incubating the cells for $4 \mathrm{~h}$ with $20 \mu \mathrm{M} 4-\mathrm{HPR}$ and/or trolox $(500 \mu \mathrm{M})$.

Mitochondrial membrane potential $\left(\Delta \Psi_{m}\right)$. Cells $(200,000)$ were cultured in $1 \mathrm{ml}$ culture medium in 12-well plates and allowed to adhere overnight. Subsequently, medium was replaced by medium containing different concentrations of 4-HPR (0-20 $\mu \mathrm{M})$. After 4-h incubation with 4-HPR, medium was replaced by medium containing $10 \mu \mathrm{g} / \mathrm{ml} \mathrm{JC}-1$ (Invitrogen, Molecular Probes). After a 15-min incubation at room temperature, the cells were washed with $500 \mu 1 \mathrm{PBS}$ and the ratio of monomeric form and aggregates was measured in a microplate reader (BMG Labtech, FLUOstar Optima); excitation $485 \mathrm{~nm}$, emission $520 \mathrm{~nm}$ and $600 \mathrm{~nm}$. In addition, the mitochondrial membrane potential was measured using $4 \mu \mathrm{M}$ TMRM (Invitrogen). Fluorescence was measured with excitation A550 nm and emmision A600 nm.

Statistical analyses. Differences in viability between $M Y C N$ amplified and MYCN single copy cell lines were analysed using the two sample t-test (SPSS 14.0.2).

\section{Results}

Effect of 4-HPR on viability, proliferation and apoptosis in neuroblastoma monolayers. A time- and concentrationdependent loss of viability and proliferation was observed in all six cell lines. The $\mathrm{IC}_{50}$ values of the six neuroblastoma cell lines towards 4-HPR are shown in Table I. A 6-12-fold difference was observed between the $\mathrm{IC}_{50}$ values both for cell proliferation and viability between the most sensitive (IMR32) and most resistant (NASS) cell line towards 4-HPR.
Table I. $\mathrm{IC}_{50}$ concentrations $(\mu \mathrm{M})$ of all cell lines for 4 -HPR, cells were treated $24,48,72 \mathrm{~h}$.

\begin{tabular}{lrrrrrrr}
\hline & \multicolumn{3}{c}{ Viability } & & \multicolumn{3}{c}{ Proliferation } \\
Cell lines & $24 \mathrm{~h}$ & $48 \mathrm{~h}$ & $72 \mathrm{~h}$ & & $24 \mathrm{~h}$ & $48 \mathrm{~h}$ & $72 \mathrm{~h}$ \\
\hline FISK & 10.0 & 5.0 & 4.3 & & 9.5 & 3.1 & 3.7 \\
SY5Y & 10.2 & 4.7 & 4.1 & & 4.0 & 3.3 & 2.0 \\
NASS & 34.0 & 11.5 & 8.3 & & 11.8 & 10.8 & 8.8 \\
SJ8 $^{\mathrm{a}}$ & 7.7 & 5.9 & 5.3 & & 5.1 & 1.9 & 0.9 \\
SJNB10 $^{\text {a }}$ & 8.6 & 4.8 & 4.6 & & 8.4 & 4.4 & 3.7 \\
IMR32 $^{\mathrm{a}}$ & 3.7 & 1.0 & 1.0 & & 2.0 & 1.1 & 0.7 \\
Mean & 12.4 & 5.5 & 4.6 & & 6.7 & 2.7 & 2.1 \\
\hline
\end{tabular}

${ }^{\mathrm{a}} \mathrm{MYCN}$ amplified cell lines.

No significant differences in sensitivity towards 4-HPR were observed between $M Y C N$ amplified and MYCN single copy cell lines.

To investigate whether this decrease in viability and proliferation was associated with apoptosis, cell cycle studies with 4-HPR treated cells were performed (Fig. 1). A substantial amount of cells in sub-G1 phase were detected after incubation with 4-HPR, which indicates DNA condensation and thus apoptosis. No cell cycle arrest was observed. In addition to cell cycle experiments, the presence or absence of cleaved PARP was studied. In all cell lines, $50 \%$ of PARP was cleaved at a 4-HPR concentration lower or equal compared to the $\mathrm{IC}_{50}$ concentration derived from the viability and the proliferation assay (data not shown).

Effect of 4-HPR on neuroblastoma spheroids. Spheroids grown from each cell line were treated for three weeks with a concentration range of 4-HPR $(0-10 \mu \mathrm{M})$. The spheroid size was monitored weekly by photo-imaging. The cross-sectional area of untreated spheroids increased exponentially in time. In contrast, spheroids showed a complete cytostasis when treated with high 4-HPR concentrations (Fig. 2). The sensitivity for 4-HPR in spheroids is similar to the sensitivity observed in monolayer, with the exception of NASS, which was $\sim 10$ times more sensitive when treated as a spheroid. An unexpected finding was that in SJNB10 and SY5Y spheroids, treated with low concentrations 4-HPR, an increased area was observed with respect to the untreated control spheroids (Fig. 2B).

Microscopical examination of H\&E stained sections of untreated IMR32 spheroids showed an organized pattern of an outer proliferative rim and an inner apoptotic core. In the rim of untreated spheroids rosettes were visible, which are histologically typical for neuroblastoma (Fig. 3A). Apoptosis was observed by the presence of both picnosis and fragmented nuclei. In 4-HPR treated spheroids, a looser cell-cell structure and a decreased amount of cells were observed (Fig. 3A). Immunohistochemical sections of IMR32 spheroids were stained with an apoptotic marker, PARP, and a proliferation marker, Ki-67. Treatment with 4-HPR (10-20 $\mu \mathrm{M}$, 

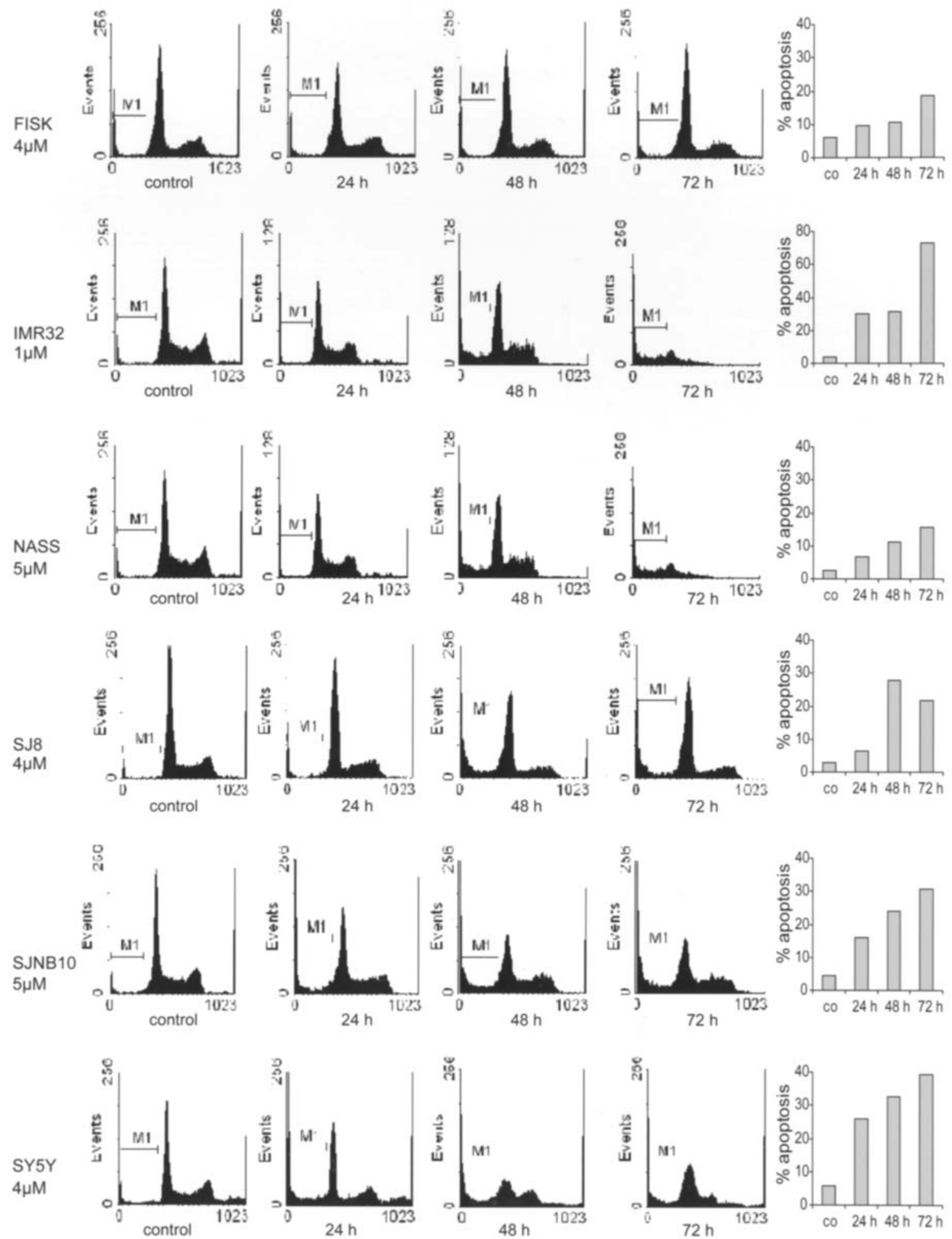

Figure 1. Analysis of apoptosis induced by 4-HPR. FACS analysis of PI stained cells; M1 represents the cells accumulated in the sub-G0 phase. The bars represent the percentage of cells in sub G0. Note: the difference in y-scale for IMR32 in the right hand panel.

1 week) showed a dose-dependent decrease in proliferation (Fig. 3B) and a dose-dependent increase in apoptotic cells (Fig. 3C).

Analysis of ROS and the mitochondrial membrane potential $\left(\Delta \Psi_{m}\right)$. In all six cell lines, a dose-dependent increase of ROS production was observed from 1 until $6 \mathrm{~h}$ of incubation with 4-HPR (Fig. 4). The production of ROS increased with $482-680 \%$ after $1 \mathrm{~h}$ of treatment with 4-HPR in IMR32, SJ8, SJNB10 and SY5Y. In contrast, FISK and NASS showed only a modest increase in ROS (maximum increase was 212 and $163 \%$, respectively) after treatment with 4 -HPR. In the 
A

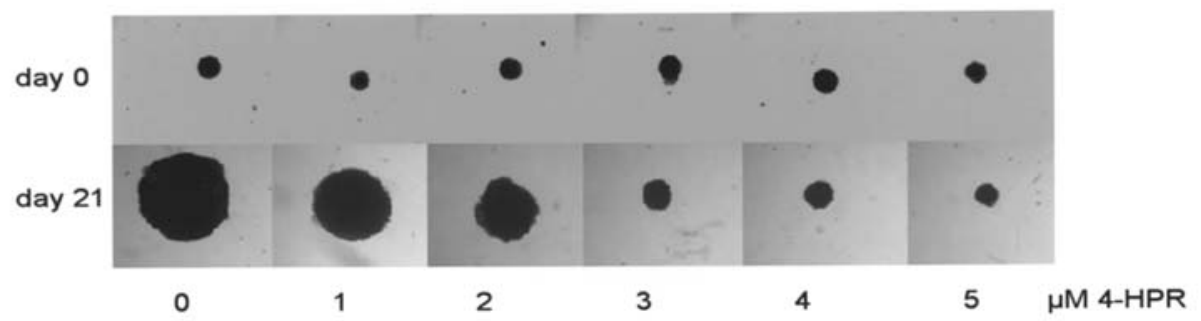

B
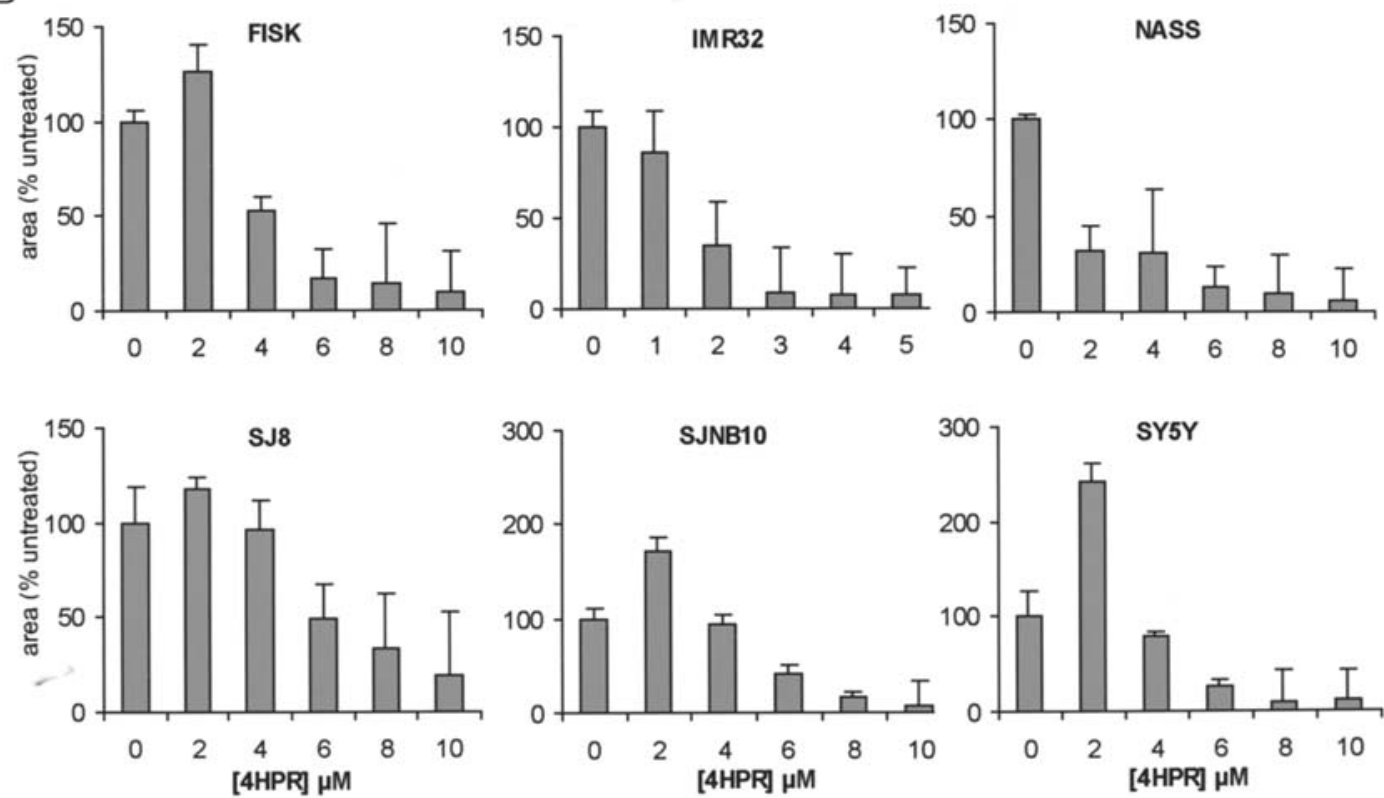

Figure 2. The effect of 4-HPR on spheroids. Spheroids were treated with 4-HPR and photographed weekly to monitor the increase of the area. A shows IMR32 spheroids treated with 4-HPR. B shows the calculated area of spheroids treated with different concentrations 4-HPR for 21 days, depicted as percentage compared to the untreated control. Each bar represents the mean area \pm SD of four experiments. IMR32 cells were treated with lower concentrations of 4HPR than the other cell lines.

presence of the antioxidant Trolox, no accumulation of 4-HPRinduced ROS occurred and trolox prevented the onset of 4HPR-induced cytotoxicity in all cell lines, except FISK (Fig. 5).

The fluorescent dye, JC-1, accumulates in mitochondria in the presence of a normal $\Delta \Psi_{\mathrm{m}}$ and forms reversible aggregates (Fig. 6A). In case of a decreased $\Delta \Psi_{\mathrm{m}}$, JC-1 aggregates dissociates into the monomeric JC-1 molecule (Fig. 6B). In all cell lines, a similar dose-dependent decrease of the $\Delta \Psi_{\mathrm{m}}$ was observed after a 4-h incubation with 4-HPR (Fig. 6C), these results were confirmed by a second probe, TMRM (data not shown). This indicates a disturbance of the $\Delta \Psi_{\mathrm{m}}$ induced by 4-HPR, which could result in increased ROS production. The decrease in $\Delta \Psi_{\mathrm{m}}$ does not correlate with the degree of ROS production.

\section{Discussion}

The synthetic retinoid 4-HPR has been shown to have a promising preventative effect in recurrence of breast cancer, because of its apoptosis-inducing effect (24). A phase I study performed in neuroblastoma showed that 4-HPR toxicity was moderate when compared to other retinoids $(25,26)$. Until now, most investigations of 4-HPR in neuroblastoma have been restricted to one or a few cell lines and only in monolayers. Due to the heterogenity of neuroblastoma, we have investigated the effectiveness of 4-HPR in vitro in a panel of six neuroblastoma cell lines. No significant difference was observed in sensitivity towards 4-HPR in MYCN amplified and MYCN single copy cell lines. This is in contrast with Reynolds et al (27) and Wei et al (28) who suggested a positive correlation between $M Y C N$ amplification and sensitivity to 4-HPR in neuroblastoma. The effective concentrations of 4-HPR inducing cytotoxicity in all cell lines were comparable with achievable plasma concentrations in vivo. In all cell lines treated with 4-HPR, apoptosis was detected by accumulation of apoptotic bodies in the sub G1-phase by flow cytometry and by PARP-cleavage. However, no cell cycle arrest was observed in 4-HPR treated neuroblastoma cells. In ovarian cancer cells, 4-HPR induced a G2 arrest which was ascribed to the formation of 4-oxo-4-HPR from 4-HPR (29). Therefore, the fact that no cell cycle arrest was observed in neuroblastoma cells might indicate that 4-HPR is not metabolized to 4-oxo4-HPR in neuroblastoma cells.

The production of ROS has been described in various tumor cell types following exposure to 4-HPR (30-32). ROS production has been measured in neuroblastoma with 4-HPR and peak production was observed after $6 \mathrm{~h}$ (17,30,33-35). We observed ROS production already after $1 \mathrm{~h}$ of 4 -HPR 

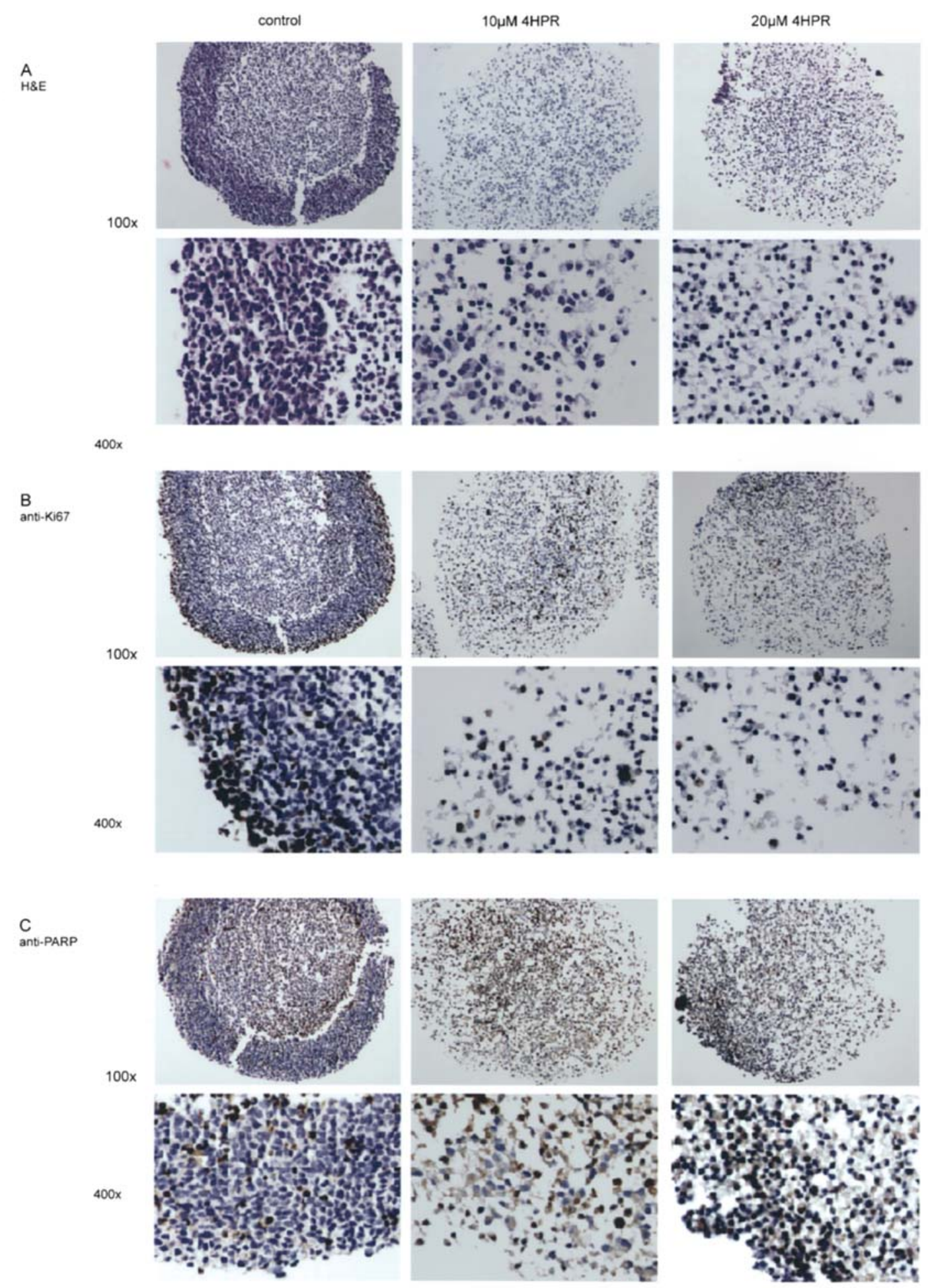

Figure 3. Histological analysis of spheroids treated with 4-HPR. IMR32 spheroids were either treated 1 week with 4-HPR (10-20 $\mu$ M) or untreated (control). Spheroids were paraffin fixed and sections were stained with: (A) H\&E for morphology. (B) Immunostaining with proliferation marker Ki-67. (C) Immunostaining with apoptosis marker cleaved PARP. All sections were examined and enlarged x100 and x400.

incubation, indicating that ROS production is an early event in response to 4-HPR incubation. Levels of ROS production correlated with the sensitivity of 4-HPR measured in the viability assays, with the exception of FISK, being sensitive to 4-HPR while producing only low levels of ROS. Trolox was able to prevent the accumulation of ROS and the loss of viability due to 4-HPR in all cell lines although the protection was suboptimal in FISK. Thus, in FISK another mechanism, apart from oxidative stress, might be responsible for the induction of apoptosis by 4-HPR. Although, the exact mechanism of ROS induction of 4-HPR is still unknown, oxidative stress appears to be one of the main apoptosis inducing mechanisms.

Since the ROS producing effect proved to be very fast it is conceivable that 4-HPR interferes directly with the mitochondrial respiratory chain (32). Apoptosis may occur 

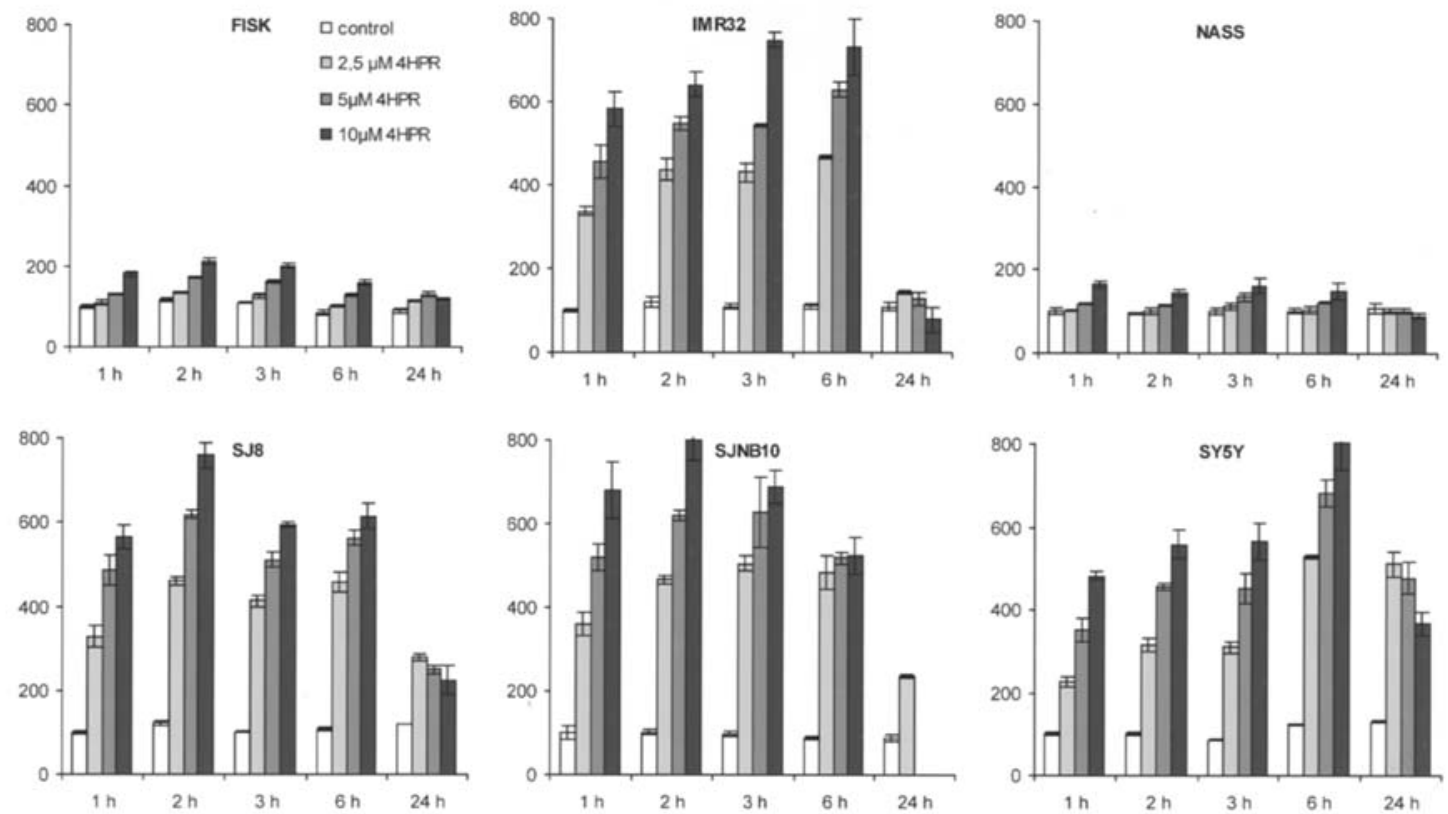

Figure 4. ROS production by 4-HPR. ROS production measured by $\mathrm{CM}-\mathrm{H}_{2} \mathrm{DCFDA}$ fluorecence in six cell lines treated with 4-HPR, depicted as percentage compared to the untreated control.

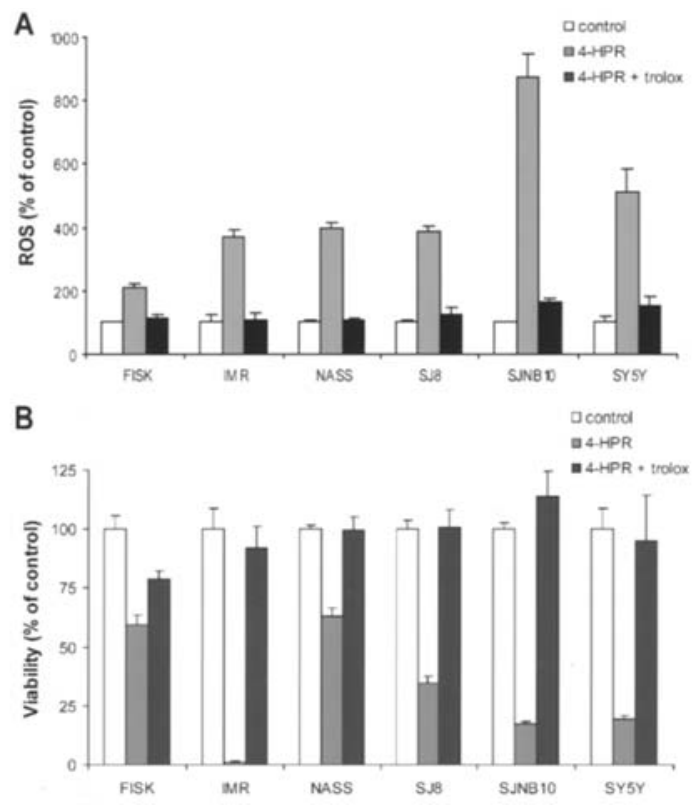

Figure 5. The effect of trolox on ROS and viability loss induced by 4-HPR. (A) ROS production measured by CM- $\mathrm{H}_{2}$ DCFDA fluorescence in cells treated with $20 \mu \mathrm{M} 4-\mathrm{HPR}$ and $500 \mu \mathrm{M}$ trolox for $4 \mathrm{~h}$, depicted as percentage of the untreated control. (B) Viability is measured using MTS-assay in cells treated with $5 \mu \mathrm{M}$ 4-HPR (NASS and SJ8 were treated with $10 \mu \mathrm{M}$ ) and $100 \mu \mathrm{M}$ trolox for $72 \mathrm{~h}$, depicted as percentage of the untreated control.

when the amount of ROS produced in the mitochondria cannot be handled by radical-scavenging antioxidants (32). In contrast, some studies suggested that ceramide and gangliosides were essential in the induction of ROS via 12LOX $(30,36,37)$. Previously, it was shown that 4-HPR did not alter the $\Delta \Psi_{\mathrm{m}}$ in the neuroblastoma cell line SY5Y (17). However, in our study a profound decrease in the $\Delta \Psi_{\mathrm{m}}$ was observed in all six cell lines after a 4-h incubation with 4-HPR.
A

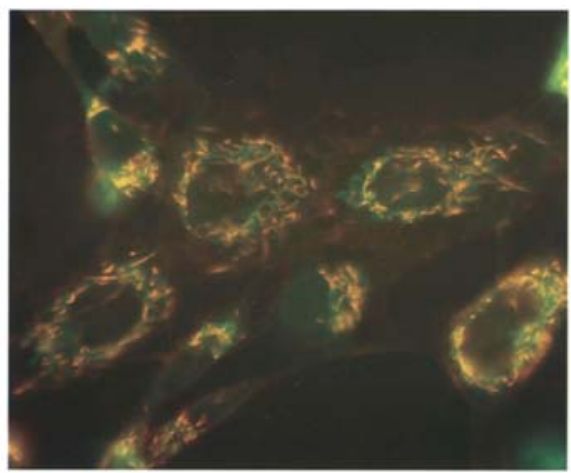

B

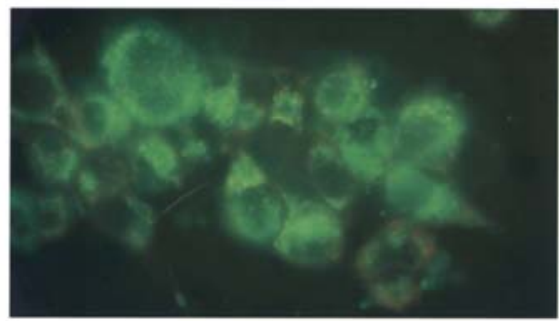

C

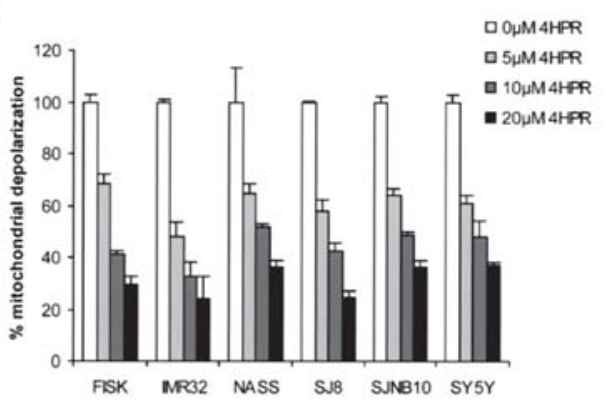

Figure 6. Changes in the mitochondrial membrane potential. (A) Representative picture of untreated NASS cells incubated with the fluorescent dye JC-1. (B) Representative picture of 4-HPR treated NASS cells incubated with the fluorescent dye JC-1. (C) Changes of the mitochondrial membrane potential was studied using the fluorescent dye, JC-1, in cells incubated $4 \mathrm{~h}$ with different 4-HPR concentrations. 
This decrease in the $\Delta \Psi_{\mathrm{m}}$ might lead to cytochrome $c$ release, and other mitochondrial enzymes, which in turn will activate caspase 9 to initiate the downstream processes of apoptosis.

For the first time, the effect of 4-HPR on multicellular tumor spheroids was studied in a panel of neuroblastoma cell lines combined with histological analysis of immunostained sections. In 4-HPR-treated spheroids of all six cell lines growth retardation and even cytostasis at higher concentrations was observed. However, SJNB10 and SY5Y spheroids treated with low concentrations $(2 \mu \mathrm{M}) 4$-HPR showed an increase in cross-sectional area compared to the controls. As shown by Marengo et al, ROS can stimulate cell proliferation when present in very low doses (38). Thus, it is conceivable that a small increase in ROS produced by low concentrations of 4-HPR might result in increased growth in these spheroids compared to controls. Our results indicated an altered cell-cell structure in 4-HPR treated spheroids. This mechanism might also explain the increased area in spheroids treated with low concentrations of 4-HPR. Nutrients might penetrate more easily due to the open structure allowing cells in the core of the spheroid to proliferate as well. Histological sections stained with an apoptosis marker, anti-cleaved-PARP and a proliferation marker, anti-Ki67 showed a concentrationdependent increase of apoptosis after 1 week of treatment with 4-HPR as well as a decrease in proliferation. Thus, the observed cytostasis (Fig. 2) is a result of decreased proliferation and induction of apoptosis and probably not a cell cycle arrest. Our results are in contrast with studies by Myatt et al who did not observe significant changes in the proliferation pattern in Ewing sarcomas (39). It is known that 4-HPR also has an anti-angiogenesis effect on neuroblastoma cells (40). In this way, 4-HPR prevents further development of micrometastases (41). Taken together, the cytotoxic effects of 4-HPR on avascular spheroids and the anti-angiogenesis effect, suggest that 4-HPR might be an effective chemotherapeutic drug for the treatment of micrometastases of different sizes.

Our investigations have shown that 4-HPR induced loss of the mitochondrial membrane potential and ROS accumulation in neuroblastoma cell lines, which is accompanied by a strong induction of apoptosis. The potency of 4-HPR to induce cytotoxicity in neuroblastoma monolayers and spheroids suggest that 4-HPR might be a promising new agent in treatment of neuroblastoma.

\section{Acknowledgements}

The authors would like to thank Dr C. Van Bree for his help with the cell cycle analysis. This study was supported by the Stichting Kindergeneeskundig Kankeronderzoek.

\section{References}

1. Seeger RC, Brodeur GM, Sather H, et al: Association of multiple copies of the $\mathrm{N}$-myc oncogene with rapid progression of neuroblastomas. N Engl J Med 313: 1111-1116, 1985.

2. Brodeur GM, Seeger RC, Sather H, et al: Clinical implications of oncogene activation in human neuroblastomas. Cancer 58: 541-545, 1986.

3. Bagatell R, Rumcheva P, London WB, et al: Outcomes of children with intermediate-risk neuroblastoma after treatment stratified by MYCN status and tumor cell ploidy. J Clin Oncol 23: 8819-8827, 2005.
4. Abemayor E and Sidell N: Human neuroblastoma cell lines as models for the in vitro study of neoplastic and neuronal cell differentiation. Environ Health Perspect 80: 3-15, 1989.

5. Villablanca JG, Khan AA, Avramis VI, et al: Phase I trial of 13cis-retinoic acid in children with neuroblastoma following bone marrow transplantation. J Clin Oncol 13: 894-901, 1995.

6. Matthay KK, Villablanca JG, Seeger RC, et al: Treatment of high-risk neuroblastoma with intensive chemotherapy, radiotherapy, autologous bone marrow transplantation, and 13-cisretinoic acid. Children's Cancer Group. N Engl J Med 341: 1165-1173, 1999.

7. Lasorella A, Iavarone A and Israel MA: Differentiation of neuroblastoma enhances $\mathrm{Bcl}-2$ expression and induces alterations of apoptosis and drug resistance. Cancer Res 55: 4711-4716, 1995.

8. Delia D, Aiello A, Lombardi L, et al: N-(4-hydroxyphenyl) retinamide induces apoptosis of malignant hemopoietic cell lines including those unresponsive to retinoic acid. Cancer Res 53: 6036-6041, 1993.

9. Mariotti A, Marcora E, Bunone G, et al: N-(4-hydroxyphenyl)retinamide: a potent inducer of apoptosis in human neuroblastoma cells. J Natl Cancer Inst 86: 1245-1247, 1994.

10. Oridate N, Lotan D, Mitchell MF, Hong WK and Lotan R: Inhibition of proliferation and induction of apoptosis in cervical carcinoma cells by retinoids: implications for chemoprevention. J Cell Biochem (Suppl) 23: 80-86, 1995.

11. Hsieh TC, Ng C and Wu JM: The synthetic retinoid N-(4hydroxyphenyl) retinamide (4-HPR) exerts antiproliferative and apoptosis-inducing effects in the androgen-independent human prostatic JCA-1 cells. Biochem Mol Biol Int 37: 499-506, 1995.

12. Clifford JL, Menter DG, Wang M, Lotan R and Lippman SM: Retinoid receptor-dependent and -independent effects of N-(4hydroxyphenyl)retinamide in F9 embryonal carcinoma cells. Cancer Res 59: 14-18, 1999.

13. Fanjul AN, Delia D, Pierotti MA, Rideout D, Yu JQ and Pfahl M: 4-Hydroxyphenyl retinamide is a highly selective activator of retinoid receptors. J Biol Chem 271: 22441-22446, 1996.

14. Sun SY, Li W, Yue P, Lippman SM, Hong WK and Lotan R: Mediation of N-(4-hydoxyphenyl)retinamide-induced apoptosis in human cancer cells by different mechanisms. Cancer Res 59: 2493-2498, 1999.

15. Lovat PE, Oliverio S, Corazzari M, et al: Bak: a downstream mediator of fenretinide-induced apoptosis of SH-SY5Y neuroblastoma cells. Cancer Res 63: 7310-7313, 2003.

16. WF Holmes, Soprano DR and Soprano KJ: Comparison of the mechanism of induction of apoptosis in ovarian carcinoma cells by the conformationally restricted synthetic retinoids CD437 and 4-HPR. J Cell Biochem 89: 262-278, 2003.

17. Lovat PE, Ranalli M, Annichiarrico-Petruzzelli M, et al: Effector mechanisms of fenretinide-induced apoptosis in neuroblastoma. Exp Cell Res 260: 50-60, 2000.

18. West GW, Weichselbaum R and Little JB: Limited penetration of methotrexate into human osteosarcoma spheroids as a proposed model for solid tumor resistance to adjuvant chemotherapy. Cancer Res 40: 3665-3668, 1980.

19. Walker KA, Mairs R, Murray T, et al: Tumor spheroid model for the biologically targeted radiotherapy of neuroblastoma micrometastases. Cancer Res 50: S1000-S1002, 1990.

20. Smith PK, Krohn RI, Hermanson GT, et al: Measurement of protein using bicinchoninic acid. Anal Biochem 150: 76-85, 1985.

21. Laemmli UK: Cleavage of structural proteins during the assembly of the head of bacteriophage T4. Nature 227: 680-685, 1970.

22. Salinovich O and Montelaro RC: Reversible staining and peptide mapping of proteins transferred to nitrocellulose after separation by sodium dodecylsulfate-polyacrylamide gel electrophoresis. Anal Biochem 156: 341-347, 1986.

23. De Ruijter AJ, Kemp S, Kramer G, et al: The novel histone deacetylase inhibitor BL1521 inhibits proliferation and induces apoptosis in neuroblastoma cells. Biochem Pharmacol 68: 1279-1288, 2004.

24. Torrisi R and Decensi A: Fenretinide and cancer prevention. Curr Oncol Rep 2: 263-270, 2000.

25. Garaventa A, Luksch R, Lo Piccolo MS, et al: Phase I trial and pharmacokinetics of fenretinide in children with neuroblastoma. Clin Cancer Res 9: 2032-2039, 2003.

26. Villablanca JG, Krailo MD, Ames MM, Reid JM, Reaman GH and Reynolds PC: Phase I trial of oral fenretinide in children with high-risk solid tumors: a report from the Children's Oncology Group (CCG 09709). J Clin Oncol 24: 3423-3430, 2006. 
27. Reynolds CP, Wang Y, Melton LJ, Einhorn PA, Slamon DJ and Maurer BJ: Retinoic-acid-resistant neuroblastoma cell lines show altered MYC regulation and high sensitivity to fenretinide. Med Pediatr Oncol 35: 597-602, 2000.

28. Wei JS, Whiteford CC, Cenacchi N, Son CG and Khan J: BBC3 mediates fenretinide-induced cell death in neuroblastoma. Oncogene 24: 7676-7683, 2005.

29. Villani MG, Appierto V, Cavadini E, et al: 4-Oxo-fenretinide, a recently identified fenretinide metabolite, induces marked G2-M cell cycle arrest and apoptosis in fenretinide-sensitive and fenretinide-resistant cell lines. Cancer Res 66: 3238-3247, 2006.

30. Maurer BJ, Metelitsa LS, Seeger RC, Cabot MC and Reynolds CP: Increase of ceramide and induction of mixed apoptosis/necrosis by $\mathrm{N}$-(4-hydroxyphenyl)-retinamide in neuroblastoma cell lines. J Natl Cancer Inst 91: 1138-1146, 1999.

31. Goto H, Takahashi H, Fujii H, Ikuta K and Yokota S: N-(4Hydroxyphenyl)retinamide (4-HPR) induces leukemia cell death via generation of reactive oxygen species. Int J Hematol 78: 219-225, 2003.

32. Suzuki S, Higuchi M, Proske RJ, Oridate N, Hong WK and Lotan R: Implication of mitochondria-derived reactive oxygen species, cytochrome $\mathrm{C}$ and caspase- 3 in $\mathrm{N}$-(4-hydroxyphenyl)retinamide-induced apoptosis in cervical carcinoma cells. Oncogene 18: 6380-6387, 1999.

33. Hewson QD, Lovat PE, Corazzari M, Catterall JB and Redfern CP: The NF-kappaB pathway mediates fenretinideinduced apoptosis in SH-SY5Y neuroblastoma cells. Apoptosis 10: 493-498, 2005.

34. Osone S, Hosoi H, Kuwahara Y, Matsumoto Y, Iehara T and Sugimoto T: Fenretinide induces sustained-activation of JNK/p38 MAPK and apoptosis in a reactive oxygen speciesdependent manner in neuroblastoma cells. Int J Cancer 112 219-224, 2004
35. Lovat PE, Ranalli M, Corazzari M, et al: Mechanisms of freeradical induction in relation to fenretinide-induced apoptosis of neuroblastoma. J Cell Biochem 89: 698-708, 2003.

36. Lovat, PE Di Sano F, Corazzari M, et al: Gangliosides link the acidic sphingomyelinase-mediated induction of ceramide to 12-lipoxygenase-dependent apoptosis of neuroblastoma in response to fenretinide. J Natl Cancer Inst 96: 1288-1299, 2004.

37. Corazzari M, Lovat PE, Oliverio S, et al: Fenretinide: a p53independent way to kill cancer cells. Biochem Biophys Res Commun 331: 810-815, 2005.

38. Marengo B, Raffaghello L, Pistoia V, et al: Reactive oxygen species: biological stimuli of neuroblastoma cell response. Cancer Lett 228: 111-116, 2005.

39. Myatt SS, Redfern CP and Burchill SA: p38MAPK-dependent sensitivity of Ewing's sarcoma family of tumors to fenretinideinduced cell death. Clin Cancer Res 11: 3136-3148, 2005.

40. Ribatti D, Raffaghello L, Marimpietri D, et al: Fenretinide as an anti-angiogenic agent in neuroblastoma. Cancer Lett 197: 181-184, 2003.

41. Ferrari N, Pfeffer U, Dell'Eva R, Ambrosini C, Noonan DM and Albini A: The transforming growth factor-beta family members bone morphogenetic protein-2 and macrophage inhibitory cytokine- 1 as mediators of the antiangiogenic activity of $\mathrm{N}-(4-$ hydroxy-phenyl)retinamide. Clin Cancer Res 11: 4610-4619, 2005. 